\title{
Preface: Association between Type 2 Diabetes and Cancer: Role of Insulin and Nitric Oxide
}

According to the World Health Organization (WHO), the prevalence of obesity across the globe has approximately doubled since 1980. In the United States, approximately one-third of the adult population is obese and an additional one-third is overweight. ${ }^{1}$ Obesity is the fastest growing lethal disease in the Western and developing countries. People do not die from obesity itself but from its complications, which shorten the lifespan. ${ }^{2,3}$ Obesity leads to many other diseases including hypercholesterolemia, type-2 diabetes (T2D), hypertension, cardiovascular disease, and cancer, which include cancers of the esophagus, colon, liver, pancreas, gallbladder, breast (postmenopausal), ovary, thyroid, prostate, multiple myeloma, kidney, and many others. ${ }^{4}$ The link between obesity and cancer is relatively underappreciated among the general population. It is estimated that as much as $20 \%$ of all cancers are due to obesity. ${ }^{5,6}$ Although the mechanisms linking increased adiposity to malignancy remain incompletely understood, growing evidence suggests that complex interactions among multiple pathways regulating steroid hormone synthesis, insulin resistance, insulin-like growth factor, adipokine and cytokine production, the immune responses, the gastrointestinal microbiota, and chronic local and systemic inflammations may collectively explain the link between overweight/obesity and carcinogenesis. ${ }^{2,7}$

This Special Issue of Critical Reviews ${ }^{\mathrm{TM}}$ in Oncogenesis, on "Association between Type 2 Diabetes and Cancer: Role of Insulin and Nitric Oxide", leads off with an article from Drs. Ghasemi and Norouzirad entitled, "Type 2 Diabetes: An Updated Overview." This article reviews glucose homeostasis in the fed and diabetic states. The article hones in on the pathogenesis of T2D as it traditionally focused on the dysfunctions of "ominous triumvirate" (liver, skeletal muscle, and $\beta$-cell) and as it has been extended to the "ominous octet," which includes defects in adipocytes (increased lipolysis), gastrointestinal tract (incretin deficiency/resistance), pancreatic $\alpha$-cells (hyperglucagonemia), kidneys (increased glucose reabsorption), and brain (insulin resistance), endothelial dysfunction, inflammation, increased oxidative stress, and hypoxia.

The next article is a review contributed by Drs. Mirmiran, Bahadoran, Ghasemi, and Hosseinpanah entitled "Type 2 Diabetes and Cancer: An Overview of Epidemiological Evidence and Potential Mechanisms." This article provides a brief overview on the epidemiological evidence supporting the association of T2D and cancer and discusses their most important potential causes. The next article entitled, "Type 2 Diabetes and Cancer: The Nitric Oxide Connection" comes from Drs. Bahadoran, Mirmiran, Ghasemi, and Kashfi. This article examines the underlying mechanisms that have been proposed as a link between T2D and cancer: hyperglycemia, hyperinsulinemia, increased levels of free steroid and peptide hormones, oxidative stress, and pro-inflammatory cytokines. Because nitric oxide (NO) can have a critical role in carcinogenesis and tumor growth progression, undesirable changes of the NO system in T2D may be an important missing link between these two pathogenic conditions. This review focuses on the hypothesis that an imbalanced NO metabolism in pathologic conditions such as T2D may contribute to carcinogenesis.

The next article is an original contribution from Drs. Pei, Lee, Khan, and Wang entitled, "Hyperactivated Insulin Signaling Cascade in Human Glioblastoma Cells." Glioblastoma multiforme (GBM) is the most common and malignant glial tumor. Although pro-growth, pro-survival, and pro-metastasis insulin signaling have been proposed to be prominent drivers of GBM progression, the insulin receptor (IR) signaling cascade in GBM has not been fully elucidated. This study directly compares insulin signaling in the U-87 MG human glioblastoma to that in primary human astrocytes. Their results show that the insulin receptor and its 
downstream signaling molecules are robustly hyperactivated, which renders a reduced insulin-induced response. These findings provide evidence for a mechanism through which GBM develops and grows aggressively even without insulin.

Drs. Bahrambeigi, Badalzadeh, Shafiei-Irannejad, and Alizadeh have reviewed the "Possible Protective Effects of Thiazolidinediones Antidiabetic Drugs in Colorectal Cancer." Thiazolidinediones (TZDs) are a class of orally available hypoglycemic medications that may be used for the treatment of T2D. They act by increasing insulin sensitivity through the peroxisome proliferator-activated receptor gamma, PPAR- $\gamma$. TZDs have antitumor effects in a wide variety of in vitro and in vivo cancer models, affecting cell cycle, apoptosis, cell differentiation and angiogenesis. This review discusses the effects of TZDs on the prevention and treatment of cancers in general with a especial emphasis on colorectal cancer. The review article entitled "The Association of Type 2 Diabetes and Site-Specific Cancers: Linking Mechanisms," was conducted by Drs. Hosseini, Alinezhad, Kharazi, and Badalzadeh. Some epidemiological studies relating to T2D and cancer are reviewed, highlighting the increased risks of both cancer incidence and mortality in patients with diabetes. Common risk factors between cancer and diabetes, possible biological links between the pathophysiology of these two diseases, and potential effects of diabetes treatment on cancer are also reviewed. Next is a systematic review by Drs. Ranjbar, Kurien, and Hall entitled "Treatment of Pediatric Overweight and Obesity: Assessing the Options." This article summarizes and assesses the efficacy of current practices in the management and treatment of pediatric overweight and obesity. Five areas of intervention most commonly used to manage and treat pediatric overweight and obesity are reviewed: physical activity, nutritional and behavioral interventions, pharmaceutical management, and surgical procedures. Finally, a review article from my laboratory with contributions by Ms. Rosen and Ms. Aslan entitled "Obesity, Type-2 Diabetes and Cancer: Mechanistic Insights" is presented. In this review, we examined the obesity-type-2diabetes-cancer relationships from a mechanistic perspective, and where appropriate, we highlighted potential pharmaceutical and dietary interventions. For the pharmaceutical interventions, we focused on metformin, TZDs, statins, and nonsteroidal anti-inflammatory drugs (NSAIDs). In each case, we delved into the mechanistic rationale of using these agents.

Since the prevalence of obesity and T2D is on the rise, we need to cultivate greater insights into the mechanisms that relate the obesity-T2D-cancer axis. Certainly there are links between obesity and T2D, between obesity and cancer, and between T2D and cancer. Collectively these are of public health interest and, hence, more clinical studies are needed to decipher these relationships. Patients that are clinically obese may require pharmacological interventions as well as lifestyle changes, and to that end, more studies are needed to access the utility of agents such as metformin, statins, NSAIDs, and TZDs in the management of such patients. It would help if BMI measurements or at least another yardstick became routinely available in general medical practice. Considering that 1.6 million deaths occur globally each year that are essentially obesity related, targeting this pathology may ultimately improve rates of cancer-related death and also rates of heart disease, stroke, and diabetes.

Guest Editor:

Khosrow Kashfi

Department of Molecular, Cellular and Biomedical Sciences

Sophie Davis School of Biomedical Education

City University of New York School of Medicine, New York, USA

Graduate Program in Biology

City University of New York Graduate Center, New York, USA

\section{REFERENCES}

1. Hales CM, Fryar CD, Carroll MD, Freedman DS, Ogden CL. Trends in obesity and severe obesity prevalence in US youth and adults by sex and age, 2007-2008 to 2015-2016. JAMA. 2018;319:1723-25.

2. Aleman JO, Eusebi LH, Ricciardiello L, Patidar K, Sanyal AJ, Holt PR. Mechanisms of obesity-induced gastrointestinal neoplasia. Gastroenterology. 2014;146:357-73. 
3. Fontaine KR, Redden DT, Wang C, Westfall AO, Allison DB. Years of life lost due to obesity. JAMA. 2003;289:187-93.

4. Lauby-Secretan B, Scoccianti C, Loomis D, Grosse Y, Bianchini F, Straif K. Body fatness and cancer-viewpoint of the IARC Working Group. N Engl J Med. 2016;375:794-98.

5. Renehan AG, Tyson M, Egger M, Heller RF, Zwahlen M. Body-mass index and incidence of cancer: a systematic review and meta-analysis of prospective observational studies. Lancet (London, UK). 2008;371:569-78.

6. Wolin KY, Carson K, Colditz GA. Obesity and cancer. Oncologist. 2010;15:556-65.

7. Gucalp A, Iyengar NM, Hudis CA, Dannenberg AJ. Targeting obesity-related adipose tissue dysfunction to prevent cancer development and progression. Semin Oncol. 2016;43:154-60. 
